\title{
Levels of zinc in plasma, erythrocytes, and hair, and levels of serum copper in patients with retinitis pigmentosa in Turkey
}

\author{
LEYLA S ATMACA, AYTEN ARCASOY, AYHAN O ÇAVDAR, \\ AND EMIN ÖZMERT
}

From the Retinal Diseases/Fluorescein Angiography/Photocoagulation Therapy Department, Eye Clinic, Ankara University

SUMMARY This study was made on 100 patients with retinitis pigmentosa and 23 of their relatives. Their plasma, erythocyte, and hair $\mathrm{Zn}$ and serum $\mathrm{Cu}$ levels were compared with those of a control group of 14 healthy persons. No statistically significant difference in these levels was found between the patients with retinitis pigmentosa and the relatives or controls.

For the past 20 years zinc has been a trace element of interest to nutritionists, medical researchers, and clinicians. Zinc has been found to be involved in many eye functions, for example, rod functions and dark adaptation. Normal rod functions and dark adaptation require that rhodopsin be produced from retinol. This in turn requires retinal reductase enzymes which have zinc as the activating constituent.

There are few studies on trace elements in retinitis pigmentosa. ${ }^{1-11}$ In 1976 Gahlot et al. ${ }^{2}$ reported on the relation between copper and zinc metabolism in retinitis pigmentosa. They measured serum copper and ceruloplasmin and 24 hour urine copper levels in retinitis pigmentosa. There are few studies on zinc metabolism in retinitis pigmentosa. In the present study, in addition to the plasma zinc and serum copper levels, we measured the erythrocyte and hair zinc levels.

\section{Material and methods}

The study was made on 100 patients with retinitis pigmentosa and their 23 healthy relatives. The results were compared with those from a control group of 14 healthy persons of similar socioeconomic class.

The group of 100 patients with retinitis pigmentosa

This study was supported by the $\mathrm{Zn}$ Deficiency Treatment Unit established by the Turkish Scientific and Technical Research Council (TUBITAK) in the Paediatric Clinic, Ankara University.

Correspondence to Leyla S Atmaca, MD, Gazi Mustafa Kemal Bulvari, 23/2, Ankara, Turkey. comprised 73 men and 27 women, the 23 relatives 18 men and 5 women, and the control group 5 men and 9 women. The age of the patients with retinitis pigmentosa ranged from 11 to 71 . The average age was 31 in the retinitis pigmentosa group, 23 in the relatives, and 18 in the controls. A complete history was obtained from all patients with retinitis pigmentosa, and medical and ophthalmological examinations were performed. In the ophthalmological examinations, in addition to routine visual tests, both eyes of all the patients were examined by Goldmann's three-mirror contact lens, Goldmann's kinetic perimetry, fluorescein angiography, and colour fundus photography.

Those who were pregnant, taking birth control medication, on other medication or suffering from any systemic disease which might effect their $\mathrm{Cu} / \mathrm{Zn}$ metabolism were eliminated

Blood and hair samples were taken from the 137 study participants by one supervised technician. All the samples were taken between 0800 and 0900 following a complete fast of at least 12 hours. The samples were transferred to the $\mathrm{Zn}$ Deficiency and Treatment Unit, where they were tested under the control of one of the authors.

The hair samples were investigated by the method suggested by Schroeder and Nason. ${ }^{12}$ The 2380 model Perkin Elmer atomic absorption spectrophotometer was used.

The statistical comparisons were made by the Barlett $\chi^{2}$ test method. Nine additional parameters 


\section{Results}

No statistically significant difference was found between the average plasma, erythrocyte, and hair zinc and serum copper levels of the patients with retinitis pigmentosa and those of the relatives and controls, nor was sex found to be a significant factor (Table 1).

The duration of the retinitis pigmentosa showed no correlation with the plasma, erythrocyte, and hair zinc and serum copper levels (Table 2).

No statistically significant difference was found between the plasma, erythrocyte, and hair zinc and serum copper levels of 21 patients with retinitis pigmentosa and those of the 23 persons who were their relatives (Table 3 ).

No statistically significant correlation was found between the average plasma, erythrocyte, and hair zinc and serum copper levels and the visual acuity evaluated in the 192 eyes of 96 patients with retinitis pigmentosa (Table 4).

Visual field defects in patients with retinitis pigmentosa were classified as mild, generalised, or tubular. The type and the quantity of the visual field defects had no correlation with the average plasma, erythrocyte, and hair zinc and serum copper levels (Table 5).

In patients with retinitis pigmentosa having additional fundus changes (such as degeneration of the

Table $1 \mathrm{Zu}$ and $\mathrm{Cu}$ levels in retinitis pigmentosa patients, unaffected relatives, and controls, mean (SEM), by sex

\begin{tabular}{|c|c|c|c|}
\hline & $\begin{array}{l}\text { Plasma } \mathrm{Zn} \\
\mu \mathrm{g} / 100 \mathrm{ml} \\
(\mathrm{SEM})\end{array}$ & $\begin{array}{l}\text { Red corpuscle } \\
\mu g / m l \\
(S E M)\end{array}$ & $\begin{array}{l}\text { Serum } C u \\
\mu g / 100 m l \\
(S E M)\end{array}$ \\
\hline \multicolumn{4}{|c|}{$R P$ patients } \\
\hline Male & $\begin{array}{c}80 \cdot 85(1.75) \\
(\mathbf{n}: 72)\end{array}$ & $\begin{array}{c}12 \cdot 24(0 \cdot 25) \\
(n: 72)\end{array}$ & $\begin{array}{l}111 \cdot 71(4 \cdot 43) \\
(n: 57)\end{array}$ \\
\hline Female & $\begin{array}{c}78 \cdot 64(3 \cdot 05) \\
(n: 24)\end{array}$ & $\begin{array}{c}12 \cdot 16(0 \cdot 34) \\
(n: 26)\end{array}$ & $\begin{array}{l}116 \cdot 73(6 \cdot 93) \\
(n: 26)\end{array}$ \\
\hline Total & $\begin{array}{c}80 \cdot 35(1 \cdot 51) \\
(n: 96)\end{array}$ & $\begin{array}{l}12 \cdot 21(0 \cdot 20) \\
(n: 98)\end{array}$ & $\begin{array}{l}113 \cdot 26(3 \cdot 72) \\
(\mathrm{n}: 83)\end{array}$ \\
\hline \multicolumn{4}{|l|}{ Relatives } \\
\hline Male & $\begin{array}{c}79 \cdot 44(3 \cdot 58) \\
\text { (n: 17) }\end{array}$ & $\begin{array}{c}12.07(0.45) \\
(n: 17)\end{array}$ & $\begin{array}{l}111.94(8 \cdot 63) \\
(n: 13)\end{array}$ \\
\hline Female & $\begin{array}{c}77 \cdot 0(4 \cdot 46) \\
(n: 5)\end{array}$ & $\begin{array}{l}11 \cdot 41(1.03) \\
(n: 5)\end{array}$ & $\begin{array}{l}131 \cdot 4(18 \cdot 23) \\
(\mathrm{n}: 5)\end{array}$ \\
\hline Total & $\begin{array}{c}78 \cdot 20(2 \cdot 38) \\
(n: 22)\end{array}$ & $\begin{array}{c}12 \cdot 20(0 \cdot 42) \\
(n: 22)\end{array}$ & $\begin{array}{l}109 \cdot 25(8 \cdot 19) \\
(n: 18)\end{array}$ \\
\hline \multicolumn{4}{|l|}{ Controls } \\
\hline Male & $\begin{array}{l}83 \cdot 8(2 \cdot 24) \\
(n: 5)\end{array}$ & $\begin{array}{l}13 \cdot 82(0 \cdot 84) \\
(n: 5)\end{array}$ & $\begin{array}{l}124 \cdot 5(24 \cdot 05) \\
(n: 4)\end{array}$ \\
\hline Female & $\begin{array}{l}77.89(4.02) \\
(n: 9)\end{array}$ & $\begin{array}{c}12.4(0.68) \\
(n: 9)\end{array}$ & $\begin{array}{l}115 \cdot 41(11 \cdot 50) \\
(n: 8)\end{array}$ \\
\hline Total & $\begin{array}{c}80 \cdot 57(3 \cdot 86) \\
(n: 14)\end{array}$ & $\begin{array}{c}12.90(0.53) \\
(n: 14)\end{array}$ & $\begin{array}{l}121 \cdot 65(10 \cdot 28) \\
\text { (n: 12) }\end{array}$ \\
\hline
\end{tabular}

$R P=$ retinitis pigmentosa. $p>0.05$ not significant.

SI conversion: $(\mu \mathrm{g} \times 10) / 100 \mathrm{ml}=\mu \mathrm{g} / \mathrm{l}$.
Table 2 Zn and $C u$ values of $R P$ patients by duration of $R P$, mean (SEM)

\begin{tabular}{llll}
\hline $\begin{array}{l}\text { Duration } \\
\text { (years) }\end{array}$ & $\begin{array}{l}\text { Plasma } \mathrm{Zn} \\
\mu \mathrm{g} / 100 \mathrm{ml} \\
(S E M)\end{array}$ & $\begin{array}{l}\text { Red corpuscle } \mathrm{Zn} \\
\mu \mathrm{g} / \mathrm{ml}\end{array}$ & $\begin{array}{l}\text { Serum } \mathrm{Cu} \\
\mu \mathrm{g} / 100 \mathrm{ml}\end{array}$ \\
& $(S E M)$ & $(S E M)$ \\
\hline$\leqslant 5$ & $82 \cdot 05(2 \cdot 62)$ & $11 \cdot 97(0 \cdot 49)$ & $112 \cdot 77(6 \cdot 80)$ \\
& $(\mathrm{n}: 20)$ & $(\mathrm{n}: 20)$ & $(\mathrm{n}: 18)$ \\
$6-10$ & $79 \cdot 55(2 \cdot 54)$ & $12 \cdot 23(0 \cdot 27)$ & $115 \cdot 70(5 \cdot 55)$ \\
& $(\mathrm{n}: 39)$ & $(\mathrm{n}: 49)$ & $(\mathrm{n}: 33)$ \\
$11-20$ & $79 \cdot 47(3 \cdot 50)$ & $12 \cdot 63(0 \cdot 46)$ & $110 \cdot 46(5 \cdot 81)$ \\
& $(\mathrm{n}: 24)$ & $(\mathrm{n}: 25)$ & $(\mathrm{n}: 20)$ \\
$>21$ & $81 \cdot 76(3 \cdot 27)$ & $11 \cdot 80(0 \cdot 64)$ & $112 \cdot 10(16 \cdot 16)$ \\
& $(\mathrm{n}: 13)$ & $(\mathrm{n}: 13)$ & $(\mathrm{n}: 12)$ \\
& & & \\
\hline
\end{tabular}

$\mathrm{RP}=$ retinitis pigmentosa. $\mathrm{p}>0.05$ not significant.

SI conversion: $(\mu \mathrm{g} \times 10) / 100 \mathrm{ml}=\mu \mathrm{g} /$.

Table $3 Z n$ and $C u$ values of RP patients compared with those of unaffected relatives, mean (SEM)

\begin{tabular}{|c|c|c|c|}
\hline & $\begin{array}{l}\text { Plasma Zn } \\
\mu g / 100 \mathrm{ml} \\
(\mathrm{SEM})\end{array}$ & $\begin{array}{l}\text { Red corpuscle } Z n \\
\mu g / m l \\
(S E M)\end{array}$ & $\begin{array}{l}\text { Serum } C u \\
\mu g / 100 \mathrm{ml} \\
\text { (SEM) }\end{array}$ \\
\hline RP patient & $\begin{array}{c}s 78 \cdot 64(3 \cdot 37) \\
(n: 21)\end{array}$ & $\begin{array}{c}11 \cdot 66(0 \cdot 41) \\
(n: 22)\end{array}$ & $\begin{array}{c}126 \cdot 10(8 \cdot 38) \\
(n: 21)\end{array}$ \\
\hline Relatives & $\begin{array}{c}78 \cdot 20(2 \cdot 38) \\
(\mathrm{n}: 22)\end{array}$ & $\begin{array}{c}12 \cdot 20(0 \cdot 42) \\
(\mathrm{n}: 22)\end{array}$ & $\begin{array}{l}109 \cdot 25(8 \cdot 19) \\
(\mathrm{n}: 18)\end{array}$ \\
\hline
\end{tabular}

$\mathrm{RP}=$ retinitis pigmentosa. $\mathrm{p}>0.05$ not significant.

SI conversion: $(\mu \mathrm{g} \times 10) / 100 \mathrm{ml}=\mu \mathrm{g} / \mathrm{l}$.

Table $4 \mathrm{Zn}$ and $\mathrm{Cu}$ values by visual acuity of eyes with $R P$, mean (SEM)

\begin{tabular}{|c|c|c|c|}
\hline $\begin{array}{l}\text { Visual } \\
\text { acuity }\end{array}$ & $\begin{array}{l}\text { Plasma } \mathrm{Zn} \\
\mu \mathrm{g} / 100 \mathrm{ml} \\
(\mathrm{SEM})\end{array}$ & $\begin{array}{l}\text { Red corpuscle } Z n \\
\mu g / m l \\
(S E M)\end{array}$ & $\begin{array}{l}\text { Serum } C u \\
\mu g / 100 \mathrm{ml} \\
(S E M)\end{array}$ \\
\hline Abs-PP & $\begin{array}{c}79.47(2.01) \\
60 \text { eyes }\end{array}$ & $\begin{array}{c}12 \cdot 79(0 \cdot 27) \\
58 \text { eyes }\end{array}$ & $\begin{array}{c}113 \cdot 58(5 \cdot 57) \\
52 \text { eyes }\end{array}$ \\
\hline $5 \mathrm{~cm} \mathrm{CF}$ & $\begin{array}{l}76 \cdot 60(2 \cdot 16) \\
46 \text { eyes }\end{array}$ & $\begin{array}{l}12 \cdot 30(0 \cdot 25) \\
50 \text { eyes }\end{array}$ & $\begin{array}{c}115 \cdot 95(5 \cdot 55) \\
43 \text { eyes }\end{array}$ \\
\hline $0 \cdot 1-0 \cdot 5$ & $\begin{array}{l}83.37(1.97) \\
60 \text { eyes }\end{array}$ & $\begin{array}{l}11 \cdot 83(0 \cdot 25) \\
61 \text { eyes }\end{array}$ & $\begin{array}{c}108 \cdot 61(4 \cdot 12) \\
46 \text { eyes }\end{array}$ \\
\hline $0 \cdot 6$ & $\begin{array}{l}75 \cdot 62(2 \cdot 04) \\
24 \text { eyes }\end{array}$ & $\begin{array}{c}12 \cdot 09(0 \cdot 48) \\
23 \text { eyes }\end{array}$ & $\begin{array}{l}117 \cdot 39(5 \cdot 19) \\
23 \text { eyes }\end{array}$ \\
\hline Total RP eyes & 192 & 196 & $166^{\circ}$ \\
\hline
\end{tabular}

$\mathrm{RP}=$ retinitis pigmentosa. $\mathrm{CF}=$ counting fingers. $\mathrm{Abs}-\mathrm{PP}=$ absolute perception and projection. $\mathrm{p}>0.05$ not significant.

SI conversion: $(\mu \mathrm{g} \times 10) / 100 \mathrm{ml}=\mu \mathrm{g} /$ l.

macula and retinal haemorrhages) $\mathrm{Zn}$ and $\mathrm{Cu}$ levels showed no statistically significant changes compared with the controls (Table 6). Likewise the anterior segment pathology in patients with retinitis pigmentosa did not influence the above mentioned $\mathrm{Zn}$ and $\mathrm{Cu}$ levels compared with the controls (Table 7).

Although the average hair $\mathrm{Zn}$ level in patients with retinitis pigmentosa was higher than that in the controls, the difference was not statistically significant (Table 8). 
Table $5 \mathrm{Zn}$ and $\mathrm{Cu}$ values by reduction in visual field, mean (SEM)

\begin{tabular}{|c|c|c|c|}
\hline $\begin{array}{l}\text { Degree of } \\
\text { constriction }\end{array}$ & $\begin{array}{l}\text { Plasma Zn } \\
\mu g / 100 \mathrm{ml} \\
(\text { SEM) }\end{array}$ & $\begin{array}{l}\text { Red corpuscle Zn } \\
\mu g / m l \\
(S E M)\end{array}$ & $\begin{array}{l}\text { Serum } C u \\
\mu g / 100 \mathrm{ml} \\
(\mathrm{SEM})\end{array}$ \\
\hline Mild & $\begin{array}{c}75 \cdot 07(2 \cdot 73) \\
\text { (n: 19) }\end{array}$ & $\begin{array}{c}11 \cdot 44(0 \cdot 44) \\
(\mathrm{n}: 22)\end{array}$ & $\begin{array}{c}116 \cdot 29(6 \cdot 34) \\
(n: 17)\end{array}$ \\
\hline Generalised & $\begin{array}{c}85 \cdot 02(4 \cdot 12) \\
(n: 19)\end{array}$ & $\begin{array}{c}11 \cdot 64(0 \cdot 37) \\
(\mathrm{n}: 20)\end{array}$ & $\begin{array}{c}120 \cdot 78(9 \cdot 75) \\
(n: 14)\end{array}$ \\
\hline Tubular & $\begin{array}{c}81 \cdot 73(3 \cdot 13) \\
(n: 17)\end{array}$ & $\begin{array}{c}12 \cdot 26(0 \cdot 53) \\
(n: 13)\end{array}$ & $\begin{array}{c}110 \cdot 30(8 \cdot 57) \\
(\mathrm{n}: 15)\end{array}$ \\
\hline
\end{tabular}

$\mathrm{p}>0.05$ not significant.

SI conversion: $(\mu \mathrm{g} \times 10) / 100 \mathrm{ml}=\mu \mathrm{g} / \mathrm{l}$.

Table 6 RP patients with additional fundus changes compared with healthy controls, mean and (SEM)

\begin{tabular}{|c|c|c|c|}
\hline & $\begin{array}{l}\text { Plasma } \mathrm{Zn} \\
\mu \mathrm{g} / 100 \mathrm{ml} \\
(\mathrm{SEM})\end{array}$ & $\begin{array}{l}\text { Red corpuscle Zn } \\
\mu \mathrm{g} / \mathrm{ml} \\
(\mathrm{SEM})\end{array}$ & $\begin{array}{l}\text { Serum } C u \\
\mu g / 100 \mathrm{ml} \\
(S E M)\end{array}$ \\
\hline \multicolumn{2}{|c|}{$\begin{array}{c}\text { RP patients } 75 \cdot 79(3 \cdot 04) \\
(n: 17)\end{array}$} & $\begin{array}{l}12 \cdot 24(0 \cdot 38) \\
(\mathrm{n}: 17)\end{array}$ & $\begin{array}{l}115 \cdot 76(7 \cdot 67) \\
(n: 16)\end{array}$ \\
\hline Controls & $\begin{array}{l}80 \cdot 57(3 \cdot 86) \\
(n: 14)\end{array}$ & $\begin{array}{l}12 \cdot 90(0 \cdot 53) \\
(n: 14)\end{array}$ & $\begin{array}{l}121 \cdot 69(10 \cdot 28) \\
\text { (n: 12) }\end{array}$ \\
\hline
\end{tabular}

$\mathbf{R P}=$ retinitis pigmentosa. $\mathrm{p}>0.05$ not significant .

SI conversion: $(\mu \mathrm{g} \times 10) / 100 \mathrm{ml}=\mu \mathrm{g} / \mathrm{l}$.

Table 7 RP patients with anterior segment pathology compared with healthy controls, mean (SEM)

\begin{tabular}{|c|c|c|c|}
\hline & $\begin{array}{l}\text { Plasma Zn } \\
\mu \mathrm{g} / 100 \mathrm{ml} \\
(\mathrm{SEM})\end{array}$ & $\begin{array}{l}\text { Red corpuscle Zn } \\
\mu g / m l \\
(S E M)\end{array}$ & $\begin{array}{l}\text { Serum } \mathrm{Cu} \\
\mu \mathrm{g} / 100 \mathrm{ml} \\
(\mathrm{SEM})\end{array}$ \\
\hline \multicolumn{2}{|c|}{$\begin{array}{c}\text { RP patients } 75 \cdot 58(1 \cdot 81) \\
(n: 42)\end{array}$} & $\begin{array}{l}12 \cdot 63(0 \cdot 32) \\
(\mathrm{n}: 41)\end{array}$ & $\begin{array}{c}112 \cdot 23(5 \cdot 87) \\
(n: 39)\end{array}$ \\
\hline Controls & $\begin{array}{l}80 \cdot 57(3 \cdot 86) \\
(n: 14)\end{array}$ & $\begin{array}{l}12 \cdot 90(0 \cdot 53) \\
(n: 14)\end{array}$ & $\begin{array}{l}121.69(10 \cdot 28) \\
\text { (n: 12) }\end{array}$ \\
\hline
\end{tabular}

$\mathbf{R P}=$ retinitis pigmentosa. $\mathrm{p}>\mathbf{0 . 0 5}$ not significant.

SI conversion: $(\mu \mathrm{g} \times 10) / 100 \mathrm{ml}=\mu \mathrm{g} /$.

Table 8 Hair $Z n$ values in RP patients and healthy controls, mean (SEM)

\begin{tabular}{ll}
\hline & Hair Zn $(\mu g / g(S E M)$ \\
\hline PR patients & $175 \cdot 63(7 \cdot 9)(\mathrm{n}: 81)$ \\
Healthy controls & $146 \cdot 34(10 \cdot 24)(\mathrm{n}: 15)$
\end{tabular}

$R P=$ retinitis pigmentosa. $p>0.05$ not significant.

\section{Discussion}

The main purpose of this study was to investigate whether any relation existed between retinitis pigmentosa and zinc metabolism. Previously only the relation of copper metabolism to retinitis pigmentosa had been extensively studied; only a few studies had been made on the relation between zinc metabolism and retinitis pigmentosa. ${ }^{19-11}$

This study showed no significant change in the average plasma, erythrocyte, and hair zinc levels in retinitis pigmentosa. In addition, the $\mathrm{Zn}$ levels showed no correlation with the duration and seriousness of the retinitis pigmentosa. Although we found an obviously low level of $\mathrm{Zn}$ in the plasma, erythrocytes, and hair in some patients with retinitis pigmentosa, this low level could not certainly be attributed to the retinitis pigmentosa, since a low level of $\mathrm{Zn}$ might have several causes.

Our findings did not confirm the studies of Gahlot et al., who had reported that disorders of copper metabolism were characteristic for retinitis pigmentosa. In 1977 Ehlers and Bülow found a normal $\mathrm{Cu}$ metabolism in patients with retinitis pigmentosa. ${ }^{3}$ This result was confirmed by Marmor et al. in 1978. ${ }^{4}$ In 1982 Mota et al. also found no change in the $\mathrm{Cu}$ metabolism in patients with retinitis pigmentosa. ${ }^{5}$

In 1981 Sangameswar $e t$ al. found a partial change in the copper metabolism of the patients with retinitis pigmentosa. ${ }^{\circ}$

We found in comparison with controls that neither serum $\mathrm{Cu}$ levels nor $\mathrm{Zn}$ metabolism in retinitis pigmentosa showed any significant changes.

\section{References}

1 Rosenthal AR, Eckhert C. Copper and zinc in ophthalmology. In: Karcioglu ZA, Sarper RM, eds. Zinc and copper in medicine. Springfield: Thomas, 1980: 579-627.

2 Gahlot DK, Khosla PK, Makashir PD, Vasuki K, Basu N, Copper metabolism in retinitis pigmentosa. Br J Ophthalmol 1976; 60: 770-4.

3 Ehlers N, Bülow N. Clinical copper metabolism parameters in patients with retinitis pigmentosa and other tapetoretinal degenerations. Br J Ophthalmol 1977; 61: 595-6.

4 Marmor MF, Nelson JW, Levin A, Copper metabolism in American retinitis pigmentosa patients. Br J Ophthalmol 1978; 62: $168-71$.

5 Mota MC, Abreu F, Marquis EP. Copper metabolism in retinitis pigmentosa. Exp Ophthalmol 1981; 7: 21-9.

6 Sangameswar SR, Satapathy M, Sitaramayya A. Copper metabolism in retinitis pigmentosa patients. Br J Ophthalmol 1981; 65: $127-30$.

7 Bastak J, Bodger J, Cinotti A, et al. Trace metals in a family with sex-linked retinitis pigmentosa. Adv Exp Med Biol 1977; 77: 43-50.

8 Crouch RK, Chambers JK. Absence of abnormal erythrocyte superoxide dismutase, copper, or zinc levels in patients with retinitis pigmentosa. $\mathrm{Br} J$ Ophthalmol 1982; 66: 417-21.

9 Karcioglu ZA, Stout R, Hahn H. Serum zinc levels in retinitis pigmentosa. Curr Eye Res 1984; 3: 1043-8.

10 Kubalska J, Sliwinska J, Skubszewska P, Krajewska-Walasek M, Pietraszek E, Pronicka E. Changes in copper and zinc levels in the serum erythrocytes and urine of patients with retinitis pigmentosa. Pediatr Pol 1984; 55: 209-16.

11 Silverstone DZ, Berson D, Seelenfreund MH. Plasma zinc levels in high myopia and retinitis pigmentosa. Metab Ophthalmol 1981; 5: 187-90.

12 Schroeder HA, Nason PA. Trace metals in human hair, $J$ Invest Dermatol 1969; 53: 71-8.

Accepted for publication 1 October 1987. 